\title{
Thiamine supplementation in patients with chronic heart failure receiving optimum medical treatment
}

\begin{abstract}
Background: Thiamine deficiency may cause heart failure and symptoms including edema, palpitation, tachycardia, weakness, malaise, and dyspnea due to water and salt retention and peripheral vasodilation. Patients with heart failure might be thiamine deficient and benefit thiamine supplementation. This study was designed to evaluate the effect of thiamin supplementation on some systolic and diastolic echocardiographic parameters and signs and symptoms of heart failure.
\end{abstract}

Methods: Fifty two patients with systolic heart failure (LVEF $<40 \%$ ) receiving optimum medical treatment, were randomized to receive $300 \mathrm{mg}$ thiamine or placebo for 1 month in a double-blind fashion (26 patients in each group). Echocardiographies were performed before and after treatment by a single investigator and sign and symptoms were evaluated (clinicaltrials.gov Identifier \# NCT01115504).

Results: Mean age was $61.44 \pm 11.8$ years and $67.3 \%$ were male. Maximal systolic tissue velocity of of septal and lateral walls (Sm waves) increased $0.99 \pm 1.46$ and $0.83 \pm 1.58 \mathrm{~cm} / \mathrm{s}$ in thiamine group and changed $0.005 \pm 1.63$ and $-0.1 \pm 1.83 \mathrm{~cm} / \mathrm{s}$ in placebo group $(\mathrm{P}=0.033$ and $\mathrm{P}=0.044$ ) respectievely. Ejection fraction or diastolic echocardiographic parameters were not influenced by thiamine supplementation and there was no significant effect on daily activities and dyspnea. However, peripheraledema improved in 9 patients $(34.6 \%)$ of thiamine group versus 1 of the placebo group $(3.8 \%)(\mathrm{P}=0.005)$.

Conclusion: In the present study thiamine supplementation had some positive effect on edema and systolic function through an increase of lateral and septal Sm. However usefulness of routine supplementation needs more study.

Keywords: thiamine, heart failure, edema, igns and symptoms, echocardiography
Volume 9 Issue 2 - 2017

\author{
Mehdi Mousavi,' Saeed Namazi, ${ }^{2}$ \\ Mohamadreza Avadi, ${ }^{3}$ Mehdi Amirahmadi, ${ }^{4}$ \\ Delara Salehifar ${ }^{5}$ \\ Interventional Cardiologist, Assistant professor, Shaheed Rajaei \\ Hospital, Alborz University of Medical Sciences, Iran \\ ${ }^{2}$ Islamic Azad University Shahroud division, Iran \\ ${ }^{3}$ Ph.D. of Pharmaceutics R\&D Manager of Hakim Pharmaceutical \\ Company Assistant professor of Nanotechnology Department, \\ Pharmaceutical Sciences Branch ,Azad university Tehran-Iran \\ ${ }^{4}$ Pharmacist, Private clinic, Iran \\ ${ }^{5}$ Student research commitee, Alborz University of Medical \\ Sciences, Iran
}

Correspondence: Mehdi Mousavi, Shaheed Rajaei Hospital, Alborz University of Medical Sciences, Karaj, Iran, Tel 0098-9123053284,Email mehdi.mousavi@mail.ncgill.ca

Received: June 16,2017 | Published: June 26, 2017

\section{Introduction}

Despite the improvement in the treatment of heart failure the mortality still remains high and it causes significant morbidity and costs worldwide. ${ }^{1}$ Thiamine (vitamin B1) is one of water soluble vitamins $^{2}$ that could be available in cereal grains, nuts, yeast and meat. ${ }^{3}$ Thiamine has an important role in cellular process and its deficiency may cause retention of sodium and water and development of vasodilation. ${ }^{3}$ Thiamine deficiency has been related to a known high cardiac output heart failure, ${ }^{1}$ traditionally known as Beriberi with manifestations including edema, palpitation, tachycardia, weakness, malaise, dyspnea, pain or parasthesia of lower extremity and decreased diastolic blood pressure with a wide pulse pressure. ${ }^{3}$

There are some evidences that heart failure patients might be thiamine deficient in comparison to healthy controls; ${ }^{4-10}$ Heart failure patients tend to have inadequate nutrient intake, ${ }^{3-5}$ on the other hand, loop ${ }^{4,5,11-16}$ and all other diuretics ${ }^{11,15}$ that may be used in heart failure may cause the hyperexcretion of thiamine,,$^{2,4,5,13-15,17}$ hence chronic diuretic treatment has been related to thiamine deficiency in a high risk patient (such as an elderly) in some $e^{2,6,11,13,17,18}$ but not all of the studies. ${ }^{719,20}$ Thiamine deficiency further impairs cardiac performance in heart failure, ${ }^{13}$ and can mimic its signs and symptoms. ${ }^{5}$ It is possible that thiamine supplementation has some useful effects in the patients with heart failure ${ }^{2,3,17}$ by prevention or correction of thiamine deficiency in heart failure, ${ }^{4,13,14}$ but this is controversial. ${ }^{2,4,7,17,21}$

Considering the low cost and minimal side-effects of thiaminereplacement therapy, and lack of randomized clinical trials regarding the favorable effect of thiamine, this prospective randomized double blind study was performed to consider the effect of thiamine supplementation on systolic and diastolic heart function and symptoms and signs of patients with heart failure.

\section{Materials/patients and methods}

Between May 2010 and Jan 2011, 52 systolic heart failure patients (LVEF $\leq 40 \%$, NYHA class II) who were routinely visited in a heart clinic (in Shahroud, Iran) and were on optimal tolerated medical therapy, were enrolled in the study. The inclusion criteria were absence of any change in clinical signs and symptoms or drug regimen of the patients in the past 3 months, due to aggravated symptoms or optimization of dosage for a survival benefit according to the present guidelines. Excluded patients were those with decompensated heart failure, bradycardia or tachycardia (needing a change in drug dosage or regimen), concomitant medical illness e.g. chronic renal failure, chronic obstructive pulmonary disease, asthma and uncontrolled hypertension.

The study was a single-site randomized placebo-controlled double blind trial and enrolled patients were randomized by means of block randomization method by a single pharmacist. Patients were assigned to randomly receive $300 \mathrm{mg}$ daily of thiamine (HakimVitamin B1300®, Hakim pharmaceutical industry, Tehran, Iran) $(n=26$, thiamine group) or placebo (Hakim pharmaceutical industry, Tehran, Iran) ( $\mathrm{n}=26$, placebo group) for 30days in a double-blind fashion.

At randomization and at the end of study, echocardiography (Sonace 8000 EX, South Korea) was performed by a single operator in a blinded fashion. Ejection fraction was measured in 2D echocardiography in apical 4 chamber and apical 2 chamber views by means of Simpson method. systolic velocity of myocardial tissue of septal and lateral walls ( $\mathrm{Sm}$ waves) were obtained by tissue 
Doppler imaging of lateral and septal wall of the left ventricle, using pulsed Doppler echocardiography with the 2 to $3 \mathrm{~mm}$ sample volume positioned about $1 \mathrm{~cm}$ from the mitral annulus in apical 4-chamber view. Early diastolic filling velocity of the left ventricle (E wave) was obtained using ulsed Doppler echocardiography with the 2 to $3 \mathrm{~mm}$ sample volume positioned at the mitral leaflet tips with the beam aligned parallel to stream in apical 4-chamber view. ${ }^{22}$ Early diastolic myocardial tissue velocity(Ea wave) was obtained using pulsed Doppler echocardiography with the 2 to $3 \mathrm{~mm}$ sample volume positioned in the myocardium of the basal ventricular wall, about $1 \mathrm{~cm}$ from the mitral annulus from the basal septum and basal lateral walls in apical 4-chamber view. ${ }^{22} \mathrm{E}$ wave and septal and lateral Ea waves were recorded at end expiration during normal breathing. ${ }^{22}$ Septal E/ $\mathrm{Ea}$ and lateral $\mathrm{E} / \mathrm{Ea}$ were calculated as markers of diastolic function.

Weight was measured in $\mathrm{Kg}$ and body mass index was presented in $\mathrm{Kg} / \mathrm{m}^{2}$. Assessment of symptoms was done by means of a self scoring system (from 1 to 7 ) with an inter-observer error of $<5 \%$ and quality of life was assessed LAMY-7 questionnaire. Patients gave scores to"Vigorous exercise", "Light exercise", "Usual daily activities", "Dyspnea", "Chest pain", "musculoskeletal pain", "Anxiety" and "General well-being" at randomization and again after 30days of treatment. Score 1 was given if the patient felt the symptoms were terrible, score 2 if very bad, score 3 if bad, score 4 if moderate, score 5 if good, score 6 if very good and score 7 if he or she felt excellent. So the more the score the better was the situation. At the second review after 30days of therapy with thiamine or placebo the patients were asked to score to the same questionnaire and increase the scores if they believed to be better or decrease the scores if they felt worse. "Edema was objectively scored 1 to 7 at randomization and after 30 of treatment by a single experienced. Local ethical committee approved the study and informed consents were obtained.

The primary outcome of the study was an increase in dyspnea score. The secondary end points were improvement in edema or systolic function of the heart as measured by ejection fraction, lateral and septal Sm wave (measured by tissue Doppler).

\section{Statistical analysis}

Sample size calculations were based a pilot study assuming $20 \%$ improvement in dyspnea in thiamine group and $10 \%$ improvement in placebo group. In order to detect a $10 \%$ difference in the dyspnea change between active and placebo with $80 \%$ power, at a $5 \%$ twotailed significance 21 patients per group were to be enrolled in this study.

SPSS 16 was used for data analysis. Numerical variables were presented as mean \pm standard deviation and categorical variables were summarized by raw numbers and percentages. Quantitative data were compared by means of student $\mathrm{T}$ test if they showed a normal distribution and non-parametric tests if not. Qualitative data were tested by means of Chi square tests and Fisher exact test if appropriate and odds ratios are presented. Multivariate analysis was performed by linear regression (backward method) or binary logistic regression tests by backward Wald methods if appropriate.Age, sex and those variables with $p$ value of less than 0.1 in univariate analysis were included in the model. $\mathrm{P}$ values of less than 0.05 were accepted significant.

\section{Results}

Mean age of the study group was $61.44 \pm 11$.8years and $67.3 \%$ were male. Mean ejection fraction was $32.98 \pm 5.51$ percent at randomization. Ten patients (19.2\%) were treated with furosemide and the prevalence of prescription of hydrochlorothiazide, spironolactone, angiotensin converting enzyme inhibitors (ACE Inhs), angiotensin receptor blockers (ARBs), beta-adrenergic blockers and digoxin were: 7 (13.5\%), 46 (88.5\%), 10 (19.2\%), 42 (80.8\%), 44 (84.6\%), $33(63.5 \%)$ respectively. A comparison of baseline characteristics in thiamine and placebo group is shown in Table 1.

1. Scores were given to each variable from 1 to 7 .

2. Septal Sm wave: Maximal systolic velocity of myocardial tissue in tissue Doppler imaging of septal wall of the left ventricle.

3. Lateral Sm wave: Maximal systolic velocity of myocardial tissue in tissue Doppler imaging of lateral wall of the left ventricle.

4. Mitral E wave: Peak mitral inflow velocity of the early rapid filling wave.

5. Septal Ea wave: Early diastolic myocardial tissue velocity of septal wall of the left ventricle.

6. Lateral Ea wave: Early diastolic myocardial tissue velocity of lateral wall of the left ventricle.

Improvement of signs and symptoms and echocardiographic determinants of systolic and diastolic cardiac function are summarized in Table 2. Dyspnea improved in 6 patient $(23.1 \%)$ of thiamine group in comparison to $3(11.5 \%)$ of placebo group $(\mathrm{P}=0.46)$. Nine patients of thiamine group $(34.6 \%)$ versus 1 of the placebo group $(3.8 \%)$ reported improvement of edema $(\mathrm{P}=0.005$, odds ratio $=13.24,95 \%$ confidence interval $=1.53-114.29)$. Multivariate analysis showed among included variables (age, sex, spironolactone and thiamine intake) thiamine had a significant independent role in improvement of edema $(\mathrm{Ex} B=13.24,95 \%$ confidence interval $=1.53-114.29, \mathrm{P}$ $=0.019)$.

1. Septal Sm wave: Maximal systolic velocity of myocardial tissue in tissue Doppler imaging of septal wall of the left ventricle.

2. Lateral Sm wave: Maximal systolic velocity of myocardial tissue in tissue Doppler imaging of lateral wall of the left ventricle

3. Mitral E wave: Peak mitral inflow velocity of the early rapid filling wave.

4. Septal Ea wave: Early diastolic myocardial tissue velocity of septal wall of the left ventricle.

5. Lateral Ea wave: Early diastolic myocardial tissue velocity of lateral wall of the left ventricle.

Table 3 summarizes changes in different quantitative variables after 30days of thiamine or placebo administration. Thiamine did not improve any of other symptoms including, vigorous and light exercise score, usual daily activities, chest pain, musculoskeletal pain, anxiety and general well-being (Table 3). Septal and lateral Sm waves increased $0.99 \pm 1.46$ and $0.83 \pm 1.58 \mathrm{~cm} / \mathrm{s}$ in thiamine group and changed $0.05 \pm 1.63$ and $-0.1 \pm 1.83 \mathrm{~cm} / \mathrm{s}$ in placebo group respectively $(\mathrm{P}=0.033$ and $\mathrm{P}=0.044$, Table 3$)$. In multi-variate analysis among possible variables that presumed to have an influence on septal and lateral $\mathrm{Sm}$, there was a trend of increase in septal and lateral $\mathrm{Sm}$ in thiamine group $(\beta=-0.22, \mathrm{P}=0.082$ and $\beta=-0.24, \mathrm{P}=0.072$ respectively).

\section{Scores were given to each variable from 1 to 7 .}

2. Septal Sm wave: Maximal systolic velocity of myocardial tissue in tissue Doppler imaging of septal wall of the left ventricle. 
3. Lateral Sm wave: Maximal systolic velocity of myocardial tissue in tissue Doppler imaging of lateral wall of the left ventricle.

4. Mitral E wave: Peak mitral inflow velocity of the early rapid filling wave.

5. Septal Ea wave: Early diastolic myocardial tissue velocity of septal wall of the left ventricle.
6. Lateral Ea wave: Early diastolic myocardial tissue velocity of lateral wall of the left ventricle.

Considering any increases of septal and lateral Sm waves as independent variables, 19 patients of thiamine group (73.1\%) versus 12 of the placebo group (46.2\%) showed an increase in septal and lateral $\mathrm{Sm}$ waves (The results were the same for both, $\mathrm{P}=0.048$, odds ratio $=3.17,95 \%$ confidence interval $=0.99-10.1$, table 2 ).

Table I Baseline characteristics of the thiamine and placebo groups. Qualitative data are shown as number (\%) and quantitative data are shown as mean \pm standard deviation

\begin{tabular}{|c|c|c|c|}
\hline Variable & Thiamine Group & Placebo Group & P value \\
\hline Male & $16(61.5 \%)$ & $19(73.1 \%)$ & 0.38 \\
\hline History of myocardial infarction & $16(66.7 \%)$ & $16(76.2 \%)$ & 0.48 \\
\hline Furosemide & $6(23.1 \%)$ & $4(15.4 \%)$ & 0.73 \\
\hline Hydrochlorothiazide & $3(11.5 \%)$ & $4(15.4 \%)$ & I \\
\hline Spironolactone & $22(84.6 \%)$ & $24(92.3 \%)$ & 0.67 \\
\hline Angiotensin converting enzyme inhibitors & $4(15.4 \%)$ & $6(23.1 \%)$ & 0.48 \\
\hline Angiotensin receptor blockers & $22(84.6 \%)$ & $20(76.9 \%)$ & 0.48 \\
\hline Beta-adrenergic blockers & $21(80.8 \%)$ & $23(88.5 \%)$ & 0.44 \\
\hline Digoxin & 17 (65.4\%) & $16(61.5 \%)$ & 0.77 \\
\hline Age (years) & $61.92 \pm 10.73$ & $60.96 \pm 12.94$ & 0.77 \\
\hline Weight $(\mathrm{Kg})$ & $66.62 \pm 12.19$ & $70.87 \pm 13.87$ & 0.25 \\
\hline Body mass index $\left(\mathrm{Kg} / \mathrm{m}^{2}\right)$ & $25.54 \pm 4.1$ & $26.04 \pm 4.61$ & 0.68 \\
\hline Dyspnea score & $5.96 \pm 1.25$ & $5.85 \pm 1.35$ & 0.76 \\
\hline Vigorous exercise score & $2.27 \pm 1.89$ & $2.54 \pm 2.16$ & 0.69 \\
\hline Light exercise score & $5.08 \pm 1.52$ & $5.08 \pm 1.81$ & I \\
\hline Usual daily activities score & $6.8 \pm 0.98$ & $6.73 \pm 0.83$ & 0.33 \\
\hline Chest pain score & $6.12 \pm 1.34$ & $6.31 \pm 1.16$ & 0.67 \\
\hline Musculoskeletal pain score & $6.08 \pm 1.35$ & $6.38 \pm 0.75$ & 0.76 \\
\hline Anxiety score & $5.46 \pm 1.68$ & $5.23 \pm 1.66$ & 0.63 \\
\hline General well-being score & $5.5 \pm 1.03$ & $5.35 \pm 0.8$ & 0.61 \\
\hline Edema score & $5.54 \pm 1.48$ & $5.96 \pm 1.22$ & 0.36 \\
\hline Dyspnea score & $5.96 \pm 1.25$ & $5.85 \pm 1.35$ & 0.76 \\
\hline Vigorous exercise score & $2.27 \pm 1.89$ & $2.54 \pm 2.16$ & 0.69 \\
\hline Light exercise score & $5.08 \pm 1.52$ & $5.08 \pm 1.81$ & I \\
\hline Usual daily activities score & $6.8 \pm 0.98$ & $6.73 \pm 0.83$ & 0.33 \\
\hline Chest pain score & $6.12 \pm 1.34$ & $6.31 \pm 1.16$ & 0.67 \\
\hline Musculoskeletal pain score & $6.08 \pm 1.35$ & $6.38 \pm 0.75$ & 0.76 \\
\hline Ejection fraction (percent) & $32.79 \pm 5.26$ & $33.17 \pm 5.85$ & 0.81 \\
\hline Septal Sm wave $(\mathrm{cm} / \mathrm{s})$ & $6.45 \pm 1.75$ & $6.36 \pm 1.28$ & 0.84 \\
\hline Lateral Sm wave $(\mathrm{cm} / \mathrm{s})$ & $6.43 \pm 1.94$ & $7.01 \pm 1.84$ & 0.27 \\
\hline Mitral E wave/ Septal Ea wave & $14.94 \pm 10.49$ & $|3.3| \pm 7.7$ & 0.55 \\
\hline Mitral E wave/ lateral Ea wave & $13.5 \pm 10.62$ & $12.05 \pm 4.6$ & 0.63 \\
\hline Systolic blood pressure (mm Hg) & $125.4 \pm 15.6$ & $124.5 \pm 19.9$ & 0.89 \\
\hline Diastolic blood pressure $(\mathrm{mm} \mathrm{Hg})$ & $73.8 \pm 9.4$ & $70.9 \pm 9$ & 0.2 \\
\hline Heart rate (beat/min) & $68.3 \pm 10.9$ & $71.1 \pm 10.3$ & 0.34 \\
\hline
\end{tabular}

Table 2 Changes in different qualitative variables after 30 days of therapy with thiamine or placebo. Data are presented as number (\%)

\begin{tabular}{llll}
\hline Variable & Thiamine Group & Placebo Group & P value \\
\hline Any increase in weight & $10(4 I .7)$ & II (45.8) & 0.77 \\
Any improvement in dyspnea & $6(23.1)$ & $3(I I .5)$ & 0.47 \\
Any improvement in vigorous exercise & I (3.8) & $4(I 5.4)$ & 0.35 \\
Any improvement in light exercise & $8(30.8)$ & $6(23.1)$ & 0.53 \\
Any improvement in usual daily activities & 0 & 0 & 0.999 \\
Any improvement in chest pain & $8(30.8)$ & $4(I 5.4)$ & 0.19 \\
Any improvement in musculoskeletal pain & $3(I I .5)$ & $3(I I .5)$ & 0.999 \\
Any improvement in anxiety & $10(38.5)$ & $6(23.1)$ & 0.23 \\
Any improvement in general well-being & $6(23.1)$ & $3(I I .5)$ & 0.47 \\
Any improvement in edema & $9(34.6)$ & $1(3.8)$ & 0.005 \\
Any increase in ejection fraction & $5(19.2)$ & $7(26.9)$ & $0.5 I$ \\
Any increase in septal Sm wave (cm/s) & $19(73.1)$ & $12(46.2)$ & 0.05
\end{tabular}


Table Continued...

\begin{tabular}{llll}
\hline Variable & Thiamine Group & Placebo Group & P value \\
\hline Any increase in lateral Sm wave (cm/s) & $19(73.1)$ & $12(46.2)$ & 0.05 \\
Any decrease in mitral E wave/ Septal Ea wave & $9(34.6)$ & $15(57.7)$ & 0.1 \\
Any decrease in mitral E wave/ lateral Ea wave & $12(46.2)$ & $14(53.8)$ & 0.58 \\
\hline
\end{tabular}

Table 3 Changes in different quantitative variables after 30 days of therapy with thiamine or placebo

\begin{tabular}{llll}
\hline Variable & Thiamine Group (Mean \pm SD) & Placebo Group (Mean \pm SD) & P value \\
\hline Weight $(\mathrm{Kg})$ & $0.9 \pm 2.22$ & $1.44 \pm 5.86$ & 0.69 \\
Dyspnea score & $0.27 \pm 0.67$ & $-0.04 \pm 0.82$ & 0.052 \\
Vigorous exercise score & $-0.19 \pm 0.57$ & $-0.04 \pm 0.82$ & $0.1 \mathrm{I}$ \\
Light exercise score & $0.35 \pm 1.5$ & $0.12 \pm 1.45$ & 0.83 \\
Usual daily activities score & $0 \pm 0$ & $-0.04 \pm 0.2$ & 0.32 \\
Chest pain score & $0.46 \pm 1.07$ & $0.23 \pm 1.18$ & 0.25 \\
Musculoskeletal pain score & $-0.27 \pm 1.15$ & $-0.19 \pm 1.17$ & 0.5 \\
Anxiety score & $0.62 \pm 1.36$ & $0.35 \pm 1.44$ & 0.54 \\
General well-being score & $0.19 \pm 0.75$ & $-0.08 \pm 0.74$ & 0.22 \\
Edema score & $0.58 \pm 1.17$ & $-0.15 \pm 0.67$ & $-5.69 \pm 17.64$ \\
Systolic blood pressure (mm Hg) & $-6.92 \pm 14.9$ & $-2.88 \pm 7.26$ & 0.008 \\
Diastolic blood pressure (mm Hg) & $-6.15 \pm 10.23$ & $2.58 \pm 10.48$ & 0.54 \\
Heart rate (beat/min) & $1.5 \pm 10.82$ & $0.00 \pm 3.46$ & 0.25 \\
Ejection fraction (percent) & $0.87 \pm 2.44$ & $0.05 \pm 1.63$ & 0.72 \\
Septal Sm wave (cm/s) & $0.99 \pm 1.46$ & $-0.1 \pm 1.83$ & 0.64 \\
Lateral Sm wave (cm/s) & $0.83 \pm 1.58$ & $-1.35 \pm 4.48$ & 0.033 \\
Mitral E wave/ Septal Ea wave & $-1.76 \pm 7.15$ & $-I .08 \pm 4.68$ & 0.044 \\
Mitral E wave/ lateral Ea wave & $-I .58 \pm 6.53$ & & 0.82 \\
\hline
\end{tabular}

\section{Discussion}

The effect of thiamine supplementation left ventricular function is not clear in patients with heart failure. ${ }^{17}$ Seligmann and colleagues and reported that oral thiamine supplementation increased left ventricular ejection fraction in 5 heart failure patients with thiamine deficiency. ${ }^{13}$ Shimon and associates as well as Schoenenberger and coworkers reported similar results in heart failure patients with long-term diuretic therapy. ${ }^{23,24}$ Witte et al. prescribed capsules containing a combination of high-dose micronutrients including thiamine to 30 heart failure patients for 9months and reported a reduction in LV volumes and an increase in LVEF. ${ }^{25}$

In our study maximal systolic velocity of myocardial tissue of septal and lateral walls (septal and lateral Sm waves) increased in thiamine group $(\mathrm{P}=0.033$ and $\mathrm{P}=0.044$, Table 3$)$. This velocity is an indicator of ventricular systolic function ${ }^{26,27}$ and correlates well with LVEF ${ }^{27}$. However we could not show a significant increase in LVEF by thiamine supplementation in contrast to placebo $(0.87 \pm$ 2.44 percent increase in LVEF in thiamine group and no increase in placebo group $\mathrm{P}=0.64$, Table 3 ), which might be due to our limited sample size. On the other hand only $19.2 \%$ of our patients were treated with furosemide $(\leq 80 \mathrm{mg} / \mathrm{d})$ and prevalence of prescription of hydrochlorothiazide and spironolactone, were $13.5 \%$ and $88.5 \%$ respectively. Considering that spironolactone might increase thiamine level in heart failure patients on loop diuretics ${ }^{28}$ we postulated that our study group might have higher blood thiamine, that might have an effect on the possible beneficial effects of thiamine supplementation on some signs and symptoms as well as ejection fraction.

To evaluate the possible effect of thiamine supplementation, using septal and lateral E/Ea waves we could not show any significant improvement in diastolic function. E/Ea ratio which is a combination of peak mitral inflow velocity of the early rapid filling wave (E wave) and early diastolic myocardial tissue velocity (Ea wave) may have a good relationship to left ventricular filling pressure. ${ }^{26-30}$ The results of our study showed that scores of dyspnea trended to improve by thiamine supplementation. Seligmann et al noticed that symptoms related to functional capacity improved by at least one New York Heart Association class in all 6 of their studied patients treated with a 7-day course of $100 \mathrm{mg}$ twice daily intravenous thiamine. ${ }^{13}$ Dyspnea as one of the most important symptoms of heart failure seems to be correlated not only to systolic dysfunction but also to end diastolic pressure. We postulated that absence of significant improvement in scores to dyspnea or symptoms like vigorous exercise, light physical activities and usual daily activities (Table 2) in spite of significant increase in lateral and septal $\mathrm{Sm}$ in our study group could be due to the absence of significant effect of thiamine on E/Ea wave and hence diastolic function.

Our patients reported an improvement in edema with thiamine supplementation (9 patients $(\% 34.6)$ of thiamine group versus 1 (\%3.8) of placebo group ( $\mathrm{P}=0.005$, Table 2$)$, that was confirmed with multivariate analysis. This novel finding in the present study suggests that thiamine supplementation could have a positive effect on edema. It has been shown that in older heart failure patients on diuretic treatment, low dose thiamine supplementation may help to prevent the development of a sub clinical wet-beriberi ${ }^{16}$ and possibly edema. Thus thiamine could be considered as a complementary drug in the treatment of heart failure patients with edema. Weakness and malaise could occur in thiamine deficiency. ${ }^{3}$ In our study thiamine did not affect scores of general well-being. Witte and his colleagues showed a significant improvement in quality of life by prescription of micronutrients to 30 heart failure patients for 9months, ${ }^{25}$ but their regimen contained various types of micronutrients rather than thiamine alone.

Thiamine deficiency may lead to confusion and psychotic symptoms, in addition to neurological signs. ${ }^{31}$ Some physicians prescribe thiamine without clear indication to patients with anxiety symptoms. In the present study thiamine supplementation did not affect scores to anxiety and we do not recommend thiamine prescription for this purpose unless the patient is clearly thiamine deficient. Thiamine deficiency may cause sodium and water retention and peripheral 
vasodilation, palpitation, tachycardia and decrease in diastolic pressure due to a widened pulse pressure. ${ }^{3}$ Seligmann ${ }^{13}$ reported an average increase of $10 \mathrm{~mm} \mathrm{Hg}$ in systolic and diastolic blood pressure in their thiamine deficient patients by 7 days of intravenous thiamine administration. ${ }^{13}$ Shimon and associates did not report any significant change in blood pressure in thiamine group. ${ }^{23}$ In our study there was no significant change in blood pressure or heart rate of heart failure patients treated with thiamine (Table 3 ).

\section{Limitations}

Measuring thiamine levels in our patients we might lead to better understanding of some of our findings. Including more patients might let us have more powered findings. Given the physiologic importance of thiamine, further investigations aimed at determining the effect in heart failure as well as the safety and efficacy is recommended.

\section{Conclusion}

In our study thiamine supplementation had no significant effect on vigorous exercise, light exercise, usual daily activities, dyspnea, chest pain, musculoskeletal pain, anxiety and general well-being, however, it improved edema and Sm wave in echocardiography. We conclude that supplementation of thiamine might be beneficial in patients who suffer from edema, however, further studies are needed to confirm this.

\section{Acknowledgements}

Authors would like to thank for Dr. Sadafsadat Mirkarimi for her support in article review and all of the employees of Dr Amirahmadi's Pharmacy for their support in randomization.

\section{Conflicts of interest}

Author declares there are no conflicts of interest.

\section{Funding}

None.

\section{References}

1. James L Jj, Mann Dp. Clinical Assessment Of Heart Failure. In: Mann D L Bro, et al., (Eds.), Braunwald's Heart Disease A Textbook Of Cardiovascular Medicine. (10th edn), Elsevier Saunders, Philadelphia, USA. 2015. p.473-483.

2. Sica Da. Loop Diuretic Therapy, Thiamine Balance, And Heart Failure Congestive Heart Failure (Greenwich, Conn). 2007;13(4):244-247.

3. Leslie D, Gheorghiade M. Is There A Role For Thiamine Supplementation In The Management Of Heart Failure? American Heart Journal. 1996;131(6):1248-1250.

4. Hanninen Sa, Darling Pb, Sole Mj, et al. The Prevalence Of Thiamin Deficiency In Hospitalized Patients With Congestive Heart Failure. J Am Coll Cardiol. 2006;47(2):354-361.

5. Wooley Ja. Characteristics Of Thiamin And Its Relevance To The Management Of Heart Failure. Nutr Clin Pract. 2008;23(5):487-493.

6. Zenuk C, Healey J, Donnelly J, et al. Thiamine Deficiency In Congestive Heart Failure Patients Receiving Long Term Furosemide Therapy. Can J Clin Pharmacol. 2003; 10(4):184-188.

7. Pfitzenmeyer P, Guilland Jc, D'athis P, et al. Thiamine Status Of Elderly Patients With Cardiac Failure Including The Effects Of Supplementation. Int J Vitam Nutr Res . 1994;64(2):113-118.

8. Brady Ja, Rock Cl, Horneffer Mr. Thiamin Status, Diuretic Medications, And The Management Of Congestive Heart Failure. J Am Diet Assoc . 1995;95(5):541-544.
9. Keith $\mathrm{Me}$, Walsh $\mathrm{Na}$, Darling $\mathrm{Pb}$, et al. B-Vitamin Deficiency In Hospitalized Patients With Heart Failure. $J$ Am Diet Assoc. 2009;109(8):1406-1410.

10. Lourenço Bh, Vieira Lp, Macedo A, et al. Nutritional Status And Adequacy Of Energy And Nutrient Intakes Among Heart Failure Patients. Arq Bras Cardiol. 2009;93(5):541-548.

11. Suter Pm, Vetter W. Diuretics And Vitamin B1: Are Diuretics A Risk Factor For Thiamin Malnutrition? Nutr Rev. 2000;58(10):319-323.

12. Levy Wc, Soine La, Huth Mm, et al. Thiamine Deficiency In Congestive Heart Failure. Am J Med. 1992;93(6):705-706.

13. Seligmann H, Halkin H, Rauchfleisch S, et al. Thiamine Deficiency In Patients With Congestive Heart Failure Receiving Long-Term Furosemide Therapy: A Pilot Study. Am J Med. 1991;91(2):151-155.

14. Rieck J, Halkin H, Almog S, et al. Urinary Loss Of Thiamine Is Increased By Low Doses Of Furosemide In Healthy Volunteers. J Lab Clin Med. 1999;134(3):238-243.

15. Lubetsky A, Winaver J, Seligmann H, et al. Urinary Thiamine Excretion In The Rat: Effects Of Furosemide, Other Diuretics, And Volume Load. J Lab Clin Med. 1999;134(3):232-237.

16. Suter Pm, Haller J, Hany A, et al. Diuretic Use: A Risk For Subclinical Thiamine Deficiency In Elderly Patients. $J$ Nutr Health Aging. 2000;4(2):69-71.

17. Blanc P, Boussuges A. Is Thiamine Supplementation Necessary In Patient With Cardiac Insufficiency? Ann Cardiol Angeiol (Paris). 2001;50(3):160-168.

18. Kwok T, Falconer-Smith Jf, Potter Jf, et al. Thiamine Status Of Elderly Patients With Cardiac Failure. Age Ageing. 1992;21(1):67-71.

19. Yue Qy, Beermann B, Lindström B, et al. No Difference In Blood Thiamine Diphosphate Levels Between Swedish Caucasian Patients With Congestive Heart Failure Treated With Furosemide And Patients Without Heart Failure. J Intern Med. 1997;242(6):491-495.

20. Härdig L, Daae C, Dellborg M, et al. Reduced Thiamine Phosphate, But Not Thiamine Diphosphate, In Erythrocytes In Elderly Patients With Congestive Heart Failure Treated With Furosemide. J Intern Med. 2000;247(5):597-600.

21. Smithline HA. Thiamine For The Treatment Of Acute Decompensated Heart Failure. Am J Emerg Med. 2007;25(1):124-126.

22. Otto C M. Textbook Of Clinical Echocardiography. (3rd edn), Elsevier Saunders, USA. 2004. P.166-195.

23. Shimon I, Almog S, Vered Z, et al. Improved Left Ventricular Function After Thiamine Supplementation In Patients With Congestive Heart Failure Receiving Long-Term Furosemide Therapy. Am $\mathrm{J}$ Med. 1995;98(5):485-490.

24. Schoenenberger Aw, Schoenenberger-Berzins R, Der Maur Ca, et al. Thiamine Supplementation In Symptomatic Chronic Heart Failure: A Randomized, Double-Blind, Placebo-Controlled, Cross-Over Pilot Study. Clin Res Cardiol. 2012;101(3):159-164.

25. Witte Kk, Nikitin Np, Parker Ac, et al. The Effect Of Micronutrient Supplementation On Quality-Of-Life And Left Ventricular Function In Elderly Patients With Chronic Heart Failure. Eur Heart $J$. 2005;26(21):2238-2244

26. Scott D, Solomon S D, Wu J, et al. Echocardiography In: Mann Dl, et al. (Eds.), Braunwald's Heart Disease A Textbook Of Cardiovascular Medicine. (10th edn), Elsevier Saunders, Philadelphia, USA. 2015. p.179-252.

27. Feigenbaum H,Armstrong WF, Ryan T. Feigenbaum's Echocardiography. (6th edn), Lippincott Wiliams \& Wilkins, USA. 2005. p.138-159. 
28. Rocha Rm, Silva Gv, De Albuquerque Dc, et al. Influence Of Spironolactone Therapy On Thiamine Blood Levels In Patients With Heart Failure. Arq Bras Cardiol. 2008; 90(5):324-328.

29. Otto Cm. Ventricular Diastolic Filling and Function. In: Otto Cm (Ed.), Textbook of Clinical Echocardiography. (5th edn), Elsevier/Saunders, Philadelphia, USA. 2013. p. 163-189.

30. Nagueh SF, Smiseth OA, Appleton CP, et al. Recommendations For The Evaluation Of Left Ventricular Diastolic Function By Echocardiography:
An Update From The American Society Of Echocardiography And The European Association Of Cardiovascular Imaging. Journal Of The American Society Of Echocardiography : Official Publication Of The American Society Of Echocardiography. 2016;29(4):277-314.

31. Petrie Wm, Ban Ta. Vitamins In Psychiatry. Do They Have A Role? Drugs. 1985;30(1):58-65. 